\title{
OA-015 PHYLOGENETIC AND DEMOGRAPHIC CHARACTERISATION OF HIV-1 TRANSMISSION NETWORKS IN A GENERAL POPULATION COHORT IN UGANDA
}

Deogratius Ssemwanga, ${ }^{1}$ Frederick Lyagoba, ${ }^{1}$ Brian Magambo, ${ }^{1}$ Anne Kapaata, ${ }^{1}$ Joseph Kirangwa, ${ }^{1}$ Maria Nannyonjo, ${ }^{1}$ Faridah Nassolo, ${ }^{1}$ Rebecca Nsubuga, ${ }_{1}$ Gonzalo Yebra, ${ }^{2}$ Andrew Brown, ${ }^{2}$ Pontiano Kaleebu'. 'MRC-UVRI AIDS, Uganda; ${ }^{2}$ University of Edinburgh (IEB), United Kingdom

\subsection{6/bmjgh-2016-000260.23}

Background The General Population Cohort (GPC) in Southwestern Uganda is a low-risk population with low HIV incidence rates $(<1 \%)$. Despite several interventions for close to 30 years, new cases of HIV continue to emerge. We set out to use phylogenetics and patients' demographic data to understand the HIV transmission dynamics in this population to inform prevention.

Methods A total of 2049 pol sequences of participants diagnosed from 2003-2015 were included in this analysis; pol sequences were from GPC $(n=1049)$, Central Uganda $(n=800)$ and Eastern Uganda $(n=200)$. Phylogenetic analysis was used to identify transmission networks. The demographic and clinical characteristics of the transmission clusters were analysed.

Results The overall subtype distribution was: A (45\%), C (3\%), $\mathrm{D}(40 \%)$ and others (12\%). The subtype distribution by region was for GPC: A (41\%), C (2\%), D (45\%) and others (12\%). For Central: A (49\%), C (4\%), D (35\%) and others (12\%). Eastern: A (60\%), C (3\%), D (24\%) and others (13\%). We identified 233 transmission clusters (cluster size variation 2-10) that comprised of 559 (27\%) of the 2049 participants.

The majority of clusters comprised transmission pairs $(n=186)$ and triplets $(n=30)$. The majority $(\sim 60 \%)$ of the 233 clusters was from the GPC and all 13 large clusters $(\geq 5)$ were also from the GPC. A significant number of clusters $(n=25$, $11 \%$ ) was formed between individuals from different geographic locations. Participants in transmission networks were associated with high-risk sexual behaviour: low condom use, high alcohol use, and partner change even with known HIV-positives.

Conclusions The transmission networks identified among individuals from the GPC and other populations or geographic regions may imply HIV introductions from outside communities. This suggests that HIV introductions into communities are common and account for a substantial number of new infections in the GPC. HIV prevention efforts should therefore target the broader communities beyond the GPC. 